\title{
Effective-medium theory of sedimentary rocks
}

\author{
Ping Sheng \\ Exxon Research and Engineering Co., Route 22 East, Clinton Township, Annandale, New Jersey 08801
}

(Received 7 September 1989)

\begin{abstract}
A simple effective-medium theory is presented, which is shown to reproduce sedimentary rocks' striking microstructural feature of bicontinuity for the fluid and solid phases at any finite porosity. The effective medium involves three components - fluid, solid, and cement material-whose material properties are related to those of the composite through a differential effective-medium scheme. The theory provides a unified explanation for the observed correlations in both the electrical and elastic characteristics of sedimentary rocks.
\end{abstract}

\section{INTRODUCTION}

As one of nature's most abundant forms of matter, rocks are known to display a rich variety of physical characteristics. In particular, the diversity in their pore geometries is easily evident in any cursory inspection of rock sample pictures. It may seem surprising, therefore, that the electrical and elastic properties of sedimentary rocks would display the approximate correlations with porosity $\phi$ that have come to be known, respectively, as the Archie's law ${ }^{1}$ and the Wyllie equation. ${ }^{2}$ Besides their importance in geophysical exploration, these correlations suggest that in spite of the apparent disorder, rock structures nonetheless possess some unifying statistical characters. For example, Archie's law states that the electrical conductivity $\sigma$ of a fluid-filled sandstone may be expressed as $\sigma=\sigma_{f} \phi^{m}$, where $\sigma_{f}$ is the fluid conductivity, and $m \simeq 2$. Viewed in the framework of the percolation theory, Archie's law implies that sandstones are inhomogeneous composites with a structure that insures the persistence of its pore connectivity to very low porosities. The nonlinear exponent value of $m \simeq 2$ is also suggestive that only a subset of the pore space actually contributes to conduction.

In 1981, Sen, Scala, and Cohen ${ }^{3}$ pointed out that the characteristics of the Archie's law may be captured by a differential effective-medium (DEM) theory, originally proposed by Bruggeman. ${ }^{4}$ However, while the DEM theory and its generalization to the elastic case $\mathrm{s}^{5}$ are successful in explaining some aspects of the observed rock properties, yet they suffer from the crucial drawback that the theory pertains only to a microstructure whose solid component is disjoint. ${ }^{6}$ The DEM theory therefore cannot account for many of the elastic characteristics of a consolidated rock. In fact, no known effective-medium theory is able to reproduce sedimentary rock's striking microstructural feature of bicontinuity for both the fluid and solid phases at any finite porosity.

In this work, I present a three-component DEM theory in which solid grains are consolidated through the presence of a third component-cement material. By also preserving the connectivity of pore space (fluid component), the theory is thus capable of modeling the electrical and elastic properties of sedimentary rocks within a unified framework. Besides offering a consistent basis for cross correlating electrical and elastic characteristics, the theory gives an excellent account of the measured correlations between the shear wave and sonic wave velocities and predicts corrections to the Wyllie equation that are consistent with observed deviations. In addition, while cement is treated as the third component mathematically, this theory can easily model a two-component composite by setting the physical properties of the cement component as equal to that of the solid. The bicontinuity of the fluid and solid phases would still be preserved in that case. In what ensues, description of the model in Sec. II is followed by a discussion in Sec. III of the microstructure implied by the model. The calculations of the electrical and elastic properties of the model are presented in Secs. IV and V, respectively.

\section{MODEL DESCRIPTION}

In the context of dielectric properties, the differential effective-medium theory was first introduced by Brugge$\operatorname{man}^{4}$ to calculate the conductivity of a two-component composite whose structure is formed by successive iterations that may be described as follows. If we have an effective-medium theory, say for the dielectric property of a two-component medium, it can always be expressed as

$$
F\left(1-\psi, \Sigma_{1}, \psi, \Sigma_{2}, \Sigma\right)=0,
$$

where $\psi$ is the volume fraction and $\Sigma_{2}$ the complex conductivity of the second component, $1-\psi$ and $\Sigma_{1}$ denote the similar quantities of the first component, and $\Sigma$ is the effective conductivity of the composite. Here $\Sigma=\sigma$ $-i \omega \kappa / 4 \pi$, where $\sigma$ is the conductivity, $\kappa$ the dielectric constant, and $\omega$ denotes the angular frequency. Starting with a homogeneous component one, we want to replace a fraction $\Delta \psi$ by component two. The change in the effective $\Sigma$ from $\Sigma=\Sigma_{1}$ at $\psi=0$ to $\Sigma_{1}+\Delta \Sigma$ at $\psi=\Delta \psi$ may be expressed as

$$
\Delta \Sigma=-\frac{(\partial F / \partial \psi)_{\psi=0}}{(\partial F / \partial \Sigma)_{\psi=0}} \Delta \psi=g\left(\Sigma_{1}, \Sigma_{2}\right) \Delta \psi
$$

To carry out further iterations, we simply replace $\Sigma_{1}$ by $\Sigma$ of the new homogenized composite medium, and remember that now $\Delta \psi$ stands for the amount of the 
composite replaced by component one, in which only an amount $\Delta \psi_{\text {actual }}$ of component two, where $\Delta \psi_{\text {(actual) }}$ $=\left[1-\psi_{(\text {actual })}\right] \Delta \psi$, is actually added. Therefore, we should replace $\Delta \psi$ by $\Delta \psi /(1-\psi)$. The final mathematical equation is therefore

$$
\frac{d \epsilon}{g\left(\Sigma, \Sigma_{2}\right)}=\frac{d \psi}{1-\psi}
$$

which is to be integrated to the desired $\psi$ value with the initial condition of $\Sigma=\Sigma_{1}$ at $\psi=0$.

Whereas in the two-component DEM theory the component one is identified as the fluid and the component two the solid, in the new three-component DEM theory I propose that the component one, the starting phase, be composed of a mixture of fluid and cement material. In mathematical terms, the conductivity $\Sigma_{1}$ is now the solution of the effective-medium equation

$$
F\left(1-f, \Sigma_{c}, f, \Sigma_{f}, \Sigma_{1}\right)=0
$$

where $\Sigma_{c}$ and $\Sigma_{f}$ denote the cement and the fluid conductivities, respectively, and $1-f$ and $f$ their respective volume fractions in component one. The fluid volume as a fraction of the total volume, or the porosity $\phi$, is therefore given by $\phi=f(1-\psi)$. It should be noted here that while the cement material is treated as a third component mathematically, physically the theory can easily model a bicontinuous two-component composite if one sets $\Sigma_{c}=\Sigma_{2}$.

For the effective-medium theories in which the particles of the two components are spherical in shape and are treated symmetrically, i.e., with respect to the interchange of the two components and their volume fractions, a $f,(1-f)$ mixture implies a bicontinuous structure in which both components form interpenetrating infinite networks if $0.4<f<0.6 .^{8}$ For the threecomponent DEM theory, this property is especially important because on the one hand, starting with a solidlike phase and replacing it with solid grains would insure the resulting medium to be solidlike at all concentrations. On the other hand, due to the special property of the DEM theory-that the starting component always remains percolating - the connected state of the fluid phase (pore space) in the starting medium is preserved by the DEM substitutional process. Thus the resulting composite is always biconnected at all finite porosities with the solid phase consolidated by the presence of cement material. The constraint of $0.4<f<0.6$ does mean, however, that $\phi \leq 0.6$. This may even be realistic, since it is indeed rare to have consolidated rocks with $\phi$ greater than $60 \%$.

\section{MICROSTRUCTURE IMPLIED BY THE MODEL}

The function of an effective-medium theory is to describe the macroscopically averaged properties of an inhomogeneous medium. As such, its equations cannot be expected to yield an explicit description of the micro- structure for the medium. Nevertheless, one can deduce, from the derivation of the effective-medium theory, a consistent physical picture of the microstructure involved. Of course, such a picture cannot be proved to be unique, nor can quantitative measures about the implied microstructures be deduced. Yet such qualitative descriptions of the implied microstructure would still be valuable because they define the types of microstructures to which a particular effective-medium theory is applicable. It is in this spirit that I wish to discuss the implied differential effective microstructure and compare it to that of sedimentary rocks.

In the mathematical description of DEM a crucial assumption is that of medium homogeneity at every substitutional step. This assumption may be realized physically if the solid grains are successively larger in size at every replacement step so that the smaller-sized inhomogeneities of the medium would appear homogeneous on the scale of the (larger) replacing grains. ${ }^{3}$ However, in relating this picture of the two-component DEM microstructure to the actual rock structures one recognizes that the extremely large range of grain sizes implied by the DEM is usually not observed.

The introduction of cement material as the third component, which is a physically identifiable component in rocks, is seen to resolve this issue. Since the solid grains are now fused by cement, they can no longer be regarded as separately identifiable particles. Instead, a physical "grain" may mean a cluster of consolidated solid particles broken from the solid matrix at points of weakest connections. Since the smaller solid particles now serve as surface features on much larger particles, the diversity in the grain sizes as implied by the microstructure of the two-component DEM directly translates into a diversity of pore surface features, which have actually been observed in careful experiments. 9,10 The elimination of the smaller-size scales in what may be counted as a "grain" also narrows the observable grain size distribution.

It should be pointed out that this effective-medium formulation does pose an unrealistic implication. That is, whereas in real rocks such as sandstones the cement is expected to be concentrated in the solid-grain-solid-grain contact region and the fluid is expected to be concentrated in the pore region, in this theory the fluid and cement are intimately mixed. However, as shown in the following sections, this aspect of the model apparently does not affect significantly the realism of the macroscopic behaviors when compared with those of sedimentary rocks.

\section{ELECTRICAL PROPERTIES- GENERALIZED ARCHIE'S LAW}

To calculate the electrical properties for the new effective medium, we start by writing down the effective medium (EM) theory for a two-component composite. ${ }^{11,12}$ The component particles are taken to be spheroidal in shape with depolarization coefficients $L_{1}=L$ and $L_{2}=L_{3}=(1-L) / 2$. The EM theory then predicts $\Sigma$ in terms of $\Sigma_{1}, \Sigma_{2}$, and the volume fraction $\psi$ of component two, where $\Sigma$ solves the following equation: 


$$
F\left(1-\psi, \Sigma_{1}, \psi, \Sigma_{2}, \Sigma\right)=\sum_{i=1}^{3}\left(\frac{(1-\psi)\left(\Sigma-\Sigma_{1}\right)}{\left(1-L_{i}\right) \Sigma+L_{i} \Sigma_{1}}+\frac{\psi\left(\Sigma-\Sigma_{2}\right)}{\left(1-L_{i}\right) \Sigma+L_{i} \Sigma_{2}}\right)=0
$$

In accordance with the procedures for constructing the DEM as outlined earlier, the differential equation to be integrated for the value of $\Sigma$ is given by Eq. (3), where $g\left(\Sigma, \Sigma_{2}\right)$ can be obtained by differentiating $F$ as given by Eq. (2). Integration of Eq. (3) with the initial condition $\Sigma(\psi=0)=\Sigma_{1}$ yields

$$
\frac{\phi}{f}=\left(\frac{\Sigma_{1}}{\Sigma}\right]^{a}\left(\frac{\Sigma_{1}+\gamma \Sigma_{2}}{\Sigma+\gamma \Sigma_{2}}\right)^{b}\left(\frac{\Sigma-\Sigma_{2}}{\Sigma_{1}-\Sigma_{2}}\right)
$$

where

$$
\begin{aligned}
& a=3 L(1-L) /(3 L+1), \\
& b=2(3 L-1)^{2} /[(5-3 L)(1+3 L)],
\end{aligned}
$$

and

$$
\gamma=(1+3 L) /(5-3 L) .
$$

Now in the three-component DEM $\Sigma_{1}$ is obtained by the solution of Eq. (5) with $L=\frac{1}{3}$. That means

$$
\begin{aligned}
& \Sigma_{1}=\frac{1}{4}\left[N+\left(N^{2}+8 \Sigma_{c} \Sigma_{f}\right)^{1 / 2}\right], \\
& N=(2-3 f) \Sigma_{c}-(1-3 f) \Sigma_{f} .
\end{aligned}
$$

Equations (6) and (7) constitute the general equations for the calculation of complex conductivity $\Sigma$ in the threecomponent DEM model.

If we specify that solid and cement are insulating and only fluid has finite conductivity $\sigma_{f}$, then at $\omega \rightarrow 0$ we have $\Sigma_{f}=\sigma_{f}, \Sigma_{c}=\Sigma_{2}=0$. Combining Eqs. (6) and (7) in that limit gives

$$
\begin{aligned}
& \sigma=k \sigma_{f} \phi^{m}, \\
& m=(5-3 L) /\left[3\left(1-L^{2}\right)\right], \\
& k=(3 f-1) / 2 f^{m} .
\end{aligned}
$$

Equation (8a) is recognized to be in the form of generalized Archie's law. ${ }^{13}$ The two constants of the theory, $L$ and $f$, are noted to be uniquely fixed by the two parameters $m$ and $k$. Requiring $m$ to be in agreement with the Archie's value of 2 yields $L \approx 0.73$, which in turn gives $a=0.186, b=0.314$, and $\gamma=1.132$. This value of $L \simeq 0.73$ implies that the solid grains are oblate spheroids with an aspect ratio of approximately 4.5. For $m=2$, the additional requirement that $0.4<f<0.6$ means that $\frac{5}{8}<k<\frac{10}{9}$, with $f=0.5$ at $k=1$. It should be noted that, since Eq. (8) is obtained by setting $\Sigma_{c}=\Sigma_{2}=0$, i.e., the cement material is identical to that of the solid, the Archie's law does not require a distinct third component for its validity. This explains the observation of Archie behavior in fused glass beads system ${ }^{14}$ where there is no distinct "cement" material.

At finite frequencies one has to solve Eqs. (7) and (8) with the values of $a, b$, and $\gamma$ specified by Archie's exponent. Since physically $\Sigma_{c}$ may be due to the clay content, $\Sigma_{c}$ itself can be a function of $\sigma_{f}$ and $\omega$. The complex behavior that results may be shown ${ }^{15}$ to mimic the surface conductivity ${ }^{16}$ and multiple relaxation times.
The Archie's law may be understood physically as a statement that only a subset of all the pore volume is effective in electrical conduction, e.g., at $\phi=0.1$ only $10 \%$ of the pore volume is effective. The ineffective volume can come about in two ways. In the picture of the percolation theory, the explanation involves the global connectivity of the pores. The ineffective volume in this case is caused by dead ends. However, this percolation explanation has two potential problems. The first one is the existence of a finite percolation threshold. This problem may be overcome by assuming the pore space to have the shape of infinite tube or sheets. The second problem, which I regard as the main drawback, is the narrow range of the porosity values (around the percolation threshold) in which the power $m$ can deviate from 1 . For $\phi$ values that are removed from the threshold by a few percent the exponent $m$ quickly reverts to 1 . This certainly does not agree with Archie's law. In contrast, the DEM theory offers a "local" explanation for the ineffective volume, which arises from the local geometry in which the diameters of the pores are larger than the connecting throats. Since only the throats are effective in conduction, much of the pore volume is thereby wasted. This explanation has the advantage of naturally yielding no percolation threshold as well as a wide range of porosity values for the nonlinear $\sigma-\phi$ relation. In the context of the fused-grain model, Roberts and Schwartz $z^{17}$ have proposed this local mechanism as a possible explanation for Archie's law. However, in that model there is always a percolation threshold at a few percent porosity, which does not agree with Archie's law.

\section{ELASTIC PROPERTIES}

For the elastic properties of the effective medium, three parameters are needed for their description in an isotropic solid: the density $\rho$, the sonic wave modulus $\beta$, and the shear wave modulus $\mu$. The sound wave, or $p$ wave, speed is then given by $V_{p}=\sqrt{\beta / \rho}$, and the shear wave, or $s$ wave, speed is given by $V_{s}=\sqrt{\mu / \rho}$. The effectivemedium theory for predicting the effective $\beta, \mu, \rho$ in terms of $\beta_{1}, \beta_{2}, \mu_{1}, \mu_{2}, \rho_{1}, \rho_{2}$, and $\psi$ has been derived by Berryman, ${ }^{18}$ and the equations can be written as a pair of coupled equations

$$
\begin{aligned}
& G\left(1-\psi, \beta_{1}, \mu_{1}, \psi, \beta_{2}, \mu_{2}, \beta, \mu\right)=0, \\
& H\left(1-\psi, \beta_{1}, \mu_{1}, \psi, \beta_{2}, \mu_{2}, \beta, \mu\right)=0,
\end{aligned}
$$

plus $\rho=(1-\psi) \rho_{1}+\psi \rho_{2}$. The explicit forms of the functions $G$ and $H$, which depend on $L$, are given in Ref. 18 . To get the DEM equations from the effective-medium theory, we follow the same procedure as that for the electrical case. Namely, $d \beta / d \psi$ and $d \mu / d \psi$ are solved in terms of the partial derivatives of $G$ and $H$ (with respect to $\psi, \beta$, and $\mu$ ) evaluated at $\psi=0$. Schematically, we may write, $d \beta=g^{\beta}\left(\beta_{1}, \mu_{1}, \beta_{2}, \mu_{2}\right) d \psi$ and $d \mu=g^{\mu}\left(\beta_{1}, \mu_{1}, \beta_{2}, \mu_{2}\right) d \psi$. By replacing $\beta_{1}, \mu_{1}$ by $\beta, \mu$ and 
$d \psi$ by $d \psi /(1-\psi)$, one gets a pair of coupled differential equations:

$$
\begin{aligned}
& (1-\psi) \frac{d \beta}{d \psi}=g^{\beta}\left(\beta, \mu, \beta_{2}, \mu_{2}\right), \\
& (1-\psi) \frac{d \mu}{d \psi}=g^{\mu}\left(\beta, \mu, \beta_{2}, \mu_{2}\right) .
\end{aligned}
$$

In general, the form $g^{\beta, \mu}$ can be written as

$$
g^{\beta, \mu}=\beta \frac{\sum_{n, l=0}^{4}\left(A_{n, l}^{\beta, \mu} \mu_{2}+B_{n, l}^{\beta, \mu} \beta_{2}\right)\left(\frac{\mu}{\mu_{2}}\right)^{n}\left[\frac{\beta}{\mu}\right)^{l}}{\sum_{n, l=0}^{4}\left(C_{n, l} \mu_{2}+D_{n, l} \beta_{2}\right)\left(\frac{\mu}{\mu_{2}}\right)^{n}\left(\frac{\beta}{\mu}\right)^{l}},
$$

where the values of the coefficients are dependent on $L$. For $L \approx 0.73$ these values are tabulated in Table I. Equations (10a) and (10b) are to be integrated with the conditions of $\beta=\beta_{1}, \mu=\mu_{1}$ at $\psi=0$. For the three-component DEM, $\beta_{2}, \mu_{2}$ are identified with those of the solid, and $\beta_{1}, \mu_{1}$ are the solutions of Eqs. (9a) and (9b) with $L=\frac{1}{3}$ :

$$
\begin{aligned}
& \beta_{1}^{-1}=(1-f)\left[\beta_{c}+\frac{4}{3}\left(\mu_{1}-\mu_{c}\right)\right]^{-1} \\
& \quad+f\left[\beta_{f}+\frac{4}{3}\left(\mu_{1}-\mu_{f}\right)\right]^{-1} \\
& \left(\mu_{1}+J\right)^{-1}=(1-f)\left(\mu_{c}+J\right)^{-1}+f\left(\mu_{f}+J\right)^{-1}
\end{aligned}
$$

where

$$
J=\mu_{1}\left(9 \beta_{1}-4 \mu_{1}\right) /\left(6 \beta_{1}+4 \mu_{1}\right) .
$$

Equation (10) is noted to depend only on the properties of the solid, denoted as component two. The values of $\mu_{1}$ and $\beta_{1}$ as given by Eq. (11) enter only as the initial values for the integration of Eq. (10). However, mathematically, there is nothing sacred about $\psi=0$ as the starting point of integration for $\mathrm{Eq}$. (10). In fact, if $\mu_{2}$ and $\beta_{2}$ are known, sometimes it would be more convenient to use the measured $\mu$ and $\beta$ values at a particular porosity as the starting point of integration. In that case one would be integrating backwards in $\psi$ to $\psi=0$. The $\mu_{1}$ and $\beta_{1}$ values can then be viewed as directly determined by one data point. From the knowledge of $\mu_{1}$ and $\beta_{1}$ one can easily solve Eq. (11) backwards to get $\mu_{c}$ and $\beta_{c}$. This is the approach I will use in the following to fit the elastic data.

In Fig. 1 I show the data by Pickett ${ }^{19}$ on the interval travel times $V_{p}^{-1}$ and $V_{s}^{-1}$ for sandstones and dolomites. In order to carry out the calculations so as to compare with the data, I first choose the values of $\beta_{2}$ and $\mu_{2}$ for the sandstone solid grains as those for the $\alpha$ quartz,${ }^{20}$ i.e., a $V_{p} / V_{s}$ ratio of 1.5 , density $\rho_{2}=\rho_{\text {solid }}=2.65 \mathrm{~g} / \mathrm{cm}^{3}$, and $\mu_{2} \simeq 0.45 \times 10^{12}$ dynes $/ \mathrm{cm}^{2}$. These translate into a $\beta_{2} \cong 1 \times 10^{12}$ dynes $/ \mathrm{cm}^{2}$. Instead of starting at $\psi=0$ ( $\phi=0.5$ ), the integration of the coupled differential Eqs. (10a) and (10b) is begun at a measured data point of $V_{p}^{-1}=58.2 \mu \mathrm{sec} / \mathrm{ft}$ and $V_{s}^{-1}=92 \mu \mathrm{sec} / \mathrm{ft}$. The porosity of that initial data point is treated as a slightly adjustable parameter so as to put the calculated porosity range in the middle of those measured samples. The resulting calculated $V_{p}^{-1}$ versus $V_{s}^{-1}$ curve is noted to be insensitive to the exact value of the initial $\phi$ value. In this case we let the starting porosity $\phi=8 \%$ since the porosity of the measured samples ranges from $5 \%$ to $30 \% .^{19}$ The solid line that goes through the sandstone data represents the result of our numerical calculations, where

$$
\begin{aligned}
& V_{p}^{-1}=\{[\phi+2.65(1-\phi)] / \beta\}^{1 / 2}, \\
& V_{s}^{-1}=\{[\phi+2.65(1-\phi)] / \mu\}^{1 / 2},
\end{aligned}
$$

TABLE I. Coefficients in Eq. (10c) evaluated at the value of $L=0.73$. Within each group the coefficients are arranged in the following order: top line $-A^{\beta}, B^{\beta} ;$ middle line $-A^{\mu}, B^{\mu}$; bottom line $-C, D$.

\begin{tabular}{rrrrrrrrrr}
\hline \\
\hline
\end{tabular}




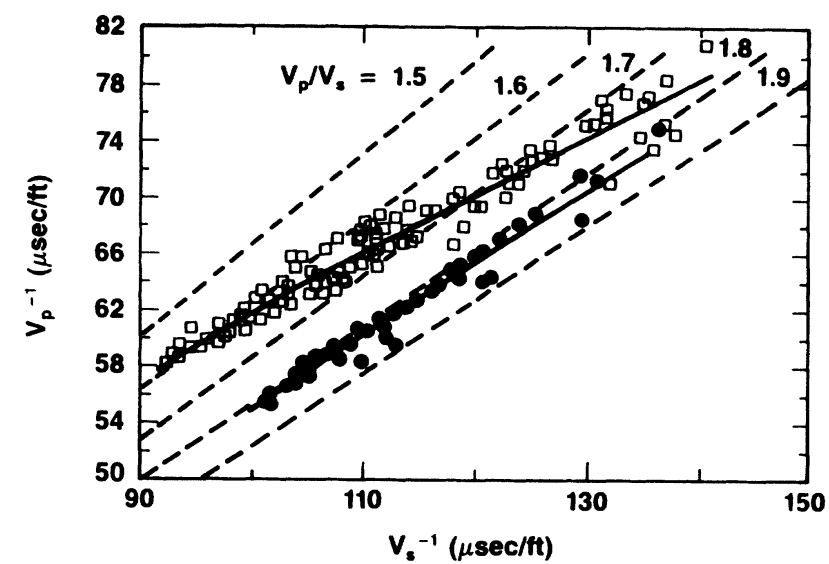

FIG. 1. Comparison between the $V_{p}^{-1}$ vs $V_{s}^{-1}$ data on sandstones and dolomites (Ref. 19) with theory, indicated by solid lines. Here the open squares denote the sandstone data, and solid circles denote the dolomite data.

and we have assumed $\rho_{c}=\rho_{\text {solid }}$. The agreement with data is striking since our result is almost indistinguishable with the curve that gives the best least-squares fit to the measured points, and we have used only one data point as the initial condition in the calculation. At $V_{s}^{-1}=140 \mu \mathrm{sec} / \mathrm{ft}$, our porosity is $21 \%$, well within the measured upper limit of $30 \%$. A similar procedure for the dolomites yields equally good agreement. In that case I have used

$$
\begin{aligned}
& \rho_{\text {solid }}=\rho_{\mathrm{c}}=2.85 \mathrm{~g} / \mathrm{cm}^{3}, \\
& \beta_{2}=1.55 \times 10^{12} \text { dynes } / \mathrm{cm}^{2}
\end{aligned}
$$

and

$$
\mu_{2}=0.46 \times 10^{12} \text { dynes } / \mathrm{cm}^{2}
$$

for the dolomite matrix. ${ }^{20}$ The beginning porosity value used is $10 \%$, and $\phi=20 \%$ at $V_{s}^{-1}=140 \mu \mathrm{sec} / \mathrm{ft}$. This again is within the range of $\phi=9-25 \%$ for the dolomite samples. In both cases the integration can be carried to $\phi=50 \%(\psi=0)$, and by solving Eq. (11) in reverse for the cement properties, given

$$
\begin{aligned}
& \beta_{1}, \mu_{1}(=\beta, \mu \text { at } \phi=0.5), \\
& \beta_{f}=2.61 \times 10^{10} \text { dynes } / \mathrm{cm}^{2}, \mu_{f}=0,
\end{aligned}
$$

and assuming $f=0.5$ as required by Archie's law, we get

$$
\begin{aligned}
& \beta_{c}=1.24 \times 10^{12} \text { dynes } / \mathrm{cm}^{2}, \\
& \mu_{c}=0.23 \times 10^{12} \text { dynes } / \mathrm{cm}^{2}
\end{aligned}
$$

for sandstones, and

$$
\begin{aligned}
& \beta_{c}=1.23 \times 10^{12} \text { dynes } / \mathrm{cm}^{2}, \\
& \mu_{c}=0.32 \times 10^{12} \text { dynes } / \mathrm{cm}^{2}
\end{aligned}
$$

for dolomites. Since the two internal parameters of the theory, $L$ and $f$, are both fixed by the electrical behavior ( $k$ and $m$ of Archie's law), the agreement with elastic characteristics may be viewed as a convincing consisten-

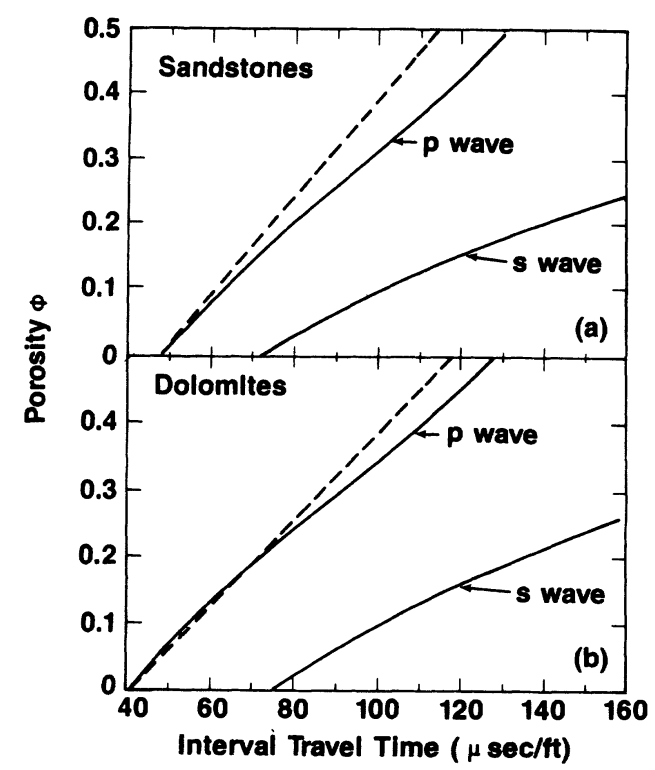

FIG. 2. Theoretically calculated porosity vs interval travel time curves for both the $p$ wave and the $s$ wave. (a) Sandstones, (b) dolomites.

cy check for the theory. It should be noted, however, that there is significant scatter in the data. This reflects the fact that while porosity and microstructure are the dominant factors affecting $V_{p}$ and $V_{s}$, they are by no means the only ones. Degree of compaction, clay content, gas saturation, etc., are all possible sources for deviations.

In Figs. 2(a) and 2(b) I show the calculated interval travel time variations with the porosity $\phi$. In the figures, the dashed lines denote the Wyllie equation, $V_{p}^{-1}$ $=(1-\phi) V_{\text {solid }}^{-1}+\phi V_{\text {fluid }}^{-1}$, where the fluid velocity is taken to be $5300 \mathrm{ft} / \mathrm{sec}$. It is seen that the Wyllie equation consistently predicts a higher porosity. This type of deviation has been summarized in the Schlumberger logging manual ${ }^{21}$ by an empirical correction factor that tends to lower the predicted porosity from a given $V_{p}^{-1}$. The present theoretical results are consistent with these empirical observations. Also, instead of calculating fluid-saturated moduli one can calculate the empty-frame moduli. Here the Wyllie equation completely breaks down because $V_{f} \cong 0$ would imply $V \simeq 0$. In this effective-medium theory, however, the $p$-wave velocity is calculated to be only 5-15\% lower than those of the fluid-saturated case. This is again in good agreement with experimental results. ${ }^{22}$

In summary, a simple three-component DEM theory is formulated and shown to yield a number of electrical and elastic behaviors that are characteristic of sedimentary rocks, thus pointing out their common microstructural origin. Further implications of the theory are presently under contemplation.

\section{ACKNOWLEDGMENTS}

I wish to thank M. Y. Zhou for the help in carrying out some of the numerical calculations and G. Winbow for pointing out Ref. 19. 
${ }^{1}$ G. E. Archie, Trans. AIME 146, 54 (1942).

${ }^{2}$ M. R. J. Wyllie, A. R. Gregory, and G. H. Gardner, Geophys. 21, 41 (1956).

${ }^{3}$ P. N. Sen, C. Scala, and M. H. Cohen, Geophys. 46, 781 (1981).

${ }^{4}$ D. A. G. Bruggeman, Ann. Phys. (Leipzig) 24, 636 (1935).

${ }^{5}$ P. Sheng and A. J. Callegari, Appl. Phys. Lett. 44, 738 (1984).

${ }^{6}$ F. Yonezawa and M. H. Cohen, J. Appl. Phys. 54, 2895 (1983);

P. Sheng and A. J. Callegari, in Physics and Chemistry of Porous Media, Proceedings of a Symposium on the Physics and Chemistry of Porous Media, AIP Conf. Proc. No. 107, edited by D. L. Johnson and P. N. Sen (AIP, New York, 1984).

${ }^{7}$ It should be noted that Biot's theory [M. A. Biot, J. Acoust. Soc. Am. 28, 168 (1956); 28, 179 (1956)], which may also be regarded as an effective-medium theory, assumes bicontinuity, but does not provide the means for calculating the phenomenological parameters (such as the frame moduli) inherent to the theory in terms of constituent properties and their microstructures.

${ }^{8}$ The $f=0.4,0.6$ limits correspond to the elastic percolation thresholds of the symmetric elastic effective-medium theory, Eq. (11).

${ }^{9}$ A. H. Thompson, A. J. Katz, and C. E. Krohn, Adv. Phys. 36, 625 (1987).
${ }^{10}$ P. Z. Wong, J. Howard, and J. S. Lin, Phys. Rev. Lett. 57, 637 (1986).

${ }^{11}$ A. N. Norris, P. Sheng, and A. J. Callegari, Appl. Phys. Lett. 57, 1990 (1985).

${ }^{12}$ K. S. Mendelson and M. H. Cohen, Geophys. 47, 257 (1982).

${ }^{13}$ L. Schwartz and S. Kimminau, Geophys. 52, 1402 (1987).

${ }^{14}$ P. Z. Wong, J. Koplik, and J. P. Tomanic, Phys. Rev. B 30, 6606 (1984).

${ }^{15}$ P. Sheng (unpublished).

${ }^{16}$ M. H. Waxman and L. J. M. Smits, Trans. AIME 243, 107 (1968).

${ }^{17}$ J. N. Roberts and L. Schwartz, Phys. Rev. B 31, 5990 (1985).

${ }^{18}$ J. G. Berryman, J. Acoust. Soc. Am. 68, 1820 (1980).

${ }^{19}$ G. R. Pickett, J. Pet. Technol. 15, 659 (1963).

${ }^{20}$ Landolt-Börnstein Series, Group V: Geophysics and Space Research, Vol. 1 of Physical Properties of Rocks, edited by G. Angenheister (Springer-Verlag, Berlin, 1982), Subvolume b, p. 33.

${ }^{21}$ Schlumberger Log Interpretation Charts (Schlumberger, Houston, 1979), p. 13.

${ }^{22}$ A. R. Gregory, in Seismic Stratigraphy-Applications to $\mathrm{Hy}$ drocarbon Exploration, edited by C. E. Payton (American Assoc. of Petroleum Geologists, Tulsa, 1977), p. 15. 